\title{
Some fixed point theorems in generating space of $b$-quasi-metric family
}

\author{
P. Sumati Kumari ${ }^{{ }^{*}}$ and Muhammad Sarwar ${ }^{2}$
}

*Correspondence:

mumy143143143@gmail.

com

${ }^{1}$ Department

of Mathematics, National

Institute of Technology-A.P,

Tadepalligudem, Andhra

Pradesh, India

Full list of author information

is available at the end of the

article

\begin{abstract}
The purpose of this work is to study some properties of "Generating space of $b$-quasimetric family"(simply $G_{b q} q^{-f a m i l y)}$ and derive some fixed point theorems using some standard contractions. Presented theorems extend and generalize many well-known results in the literature of fixed point theory.
\end{abstract}

Keywords: Generating space of b-quasi-metric family, s-h generating b-quasicontraction, s-z generating b-quasi-contraction

Mathematics Subject Classification: $47 \mathrm{H} 10,54 \mathrm{H} 25$

\section{Background}

Banach (1922) established a remarkable fixed point theorem known as the "Banach Contraction Principle." This renowned principle assures the existence and uniqueness of fixed points of certain self maps of metric spaces and gives a constructive method to find those fixed points.

(Banach 1922) Let $(X, d)$ be a complete metric space and $f: X \rightarrow X$ be a mapping, there exists a number $t, 0 \leq t<1$, such that, for each $x, y \in X$,

$$
d(f x, f y) \leq t d(x, y) .
$$

Then $f$ has a fixed point.

It is well known that Banach's contraction principle is one of the decisive result of functional analysis. A huge number of generalizations of the Banach contraction principle have appeared. Of all these, the following generalization of Kannan (1968) and Chatterjea (1972) stands at the top.

(Kannan 1968) Let $(X, d)$ be a complete metric space and $f: X \rightarrow X$ be a mapping, there exists a number $t, 0<t<\frac{1}{2}$, such that, for each $x, y \in X$,

$$
d(f x, f y) \leq t[d(x, f x)+d(y, f y)] .
$$

Then $T$ has a fixed point.

It is interesting that Kannan's fixed point theorem is very predominant because Subrahmanyam (1975) proved that, Kannan's theorem describes the completeness of the metric. In other words, a metric space $X$ is complete if and only if every Kannan mapping on $X$ has a fixed point.

(c) 2016 Kumari and Sarwar. This article is distributed under the terms of the Creative Commons Attribution 4.0 International License (http://creativecommons.org/licenses/by/4.0/), which permits unrestricted use, distribution, and reproduction in any medium, provided you give appropriate credit to the original author(s) and the source, provide a link to the Creative Commons license, and indicate if changes were made. 
(Chatterjea 1972) There exists $\alpha \in[0,1)$ such that, for all $x, y \in X$,

$$
d(f(x), f(y)) \leq \frac{\alpha}{2}[d(x, f(y))+d(y, f(x))]
$$

Then $f$ has a fixed point.

On the other hand, the traditional theory of a metric space has been generalized in wide directions. Some of such generalizations are dislocated metric spaces (Matthews 1986), dislocated quasi-metric spaces (Zeyada et al. 2006), dislocated symmetric spaces Ramabhadra et al. (2014) and quasi-symmetric spaces (Kumari and Ramana 2014) [for more new spaces and related results can be found in Bakhtin (1989), Branciari (2000), Kumari et al. (2012), Kumari et al. (2015), Kumari et al. (2015)].

In 1997, Chang et al. (1997) introduced a definition of "generating space of quasi-metric family" which is a generalization of quasi-metric space. He proved some interesting fixed point theorems and coincidence point theorems in generating space of quasi-metric family.

Later, Lee et al. (1999) define a family of weak quasi-metrics in a generating space of quasi-metric family. He proved Takahashi-type minimization theorem, a generalized Ekeland variational principle and a general Caristi-type fixed point theorem for set-valued maps in complete generating spaces of quasi-metric family by using a family of weak quasi-metrics. He also proved fixed point theorem for set-valued maps in complete generating spaces of quasi-metric family without considering of lower semi-continuity.

Very recently, Kumari and Panthi $(2015,2016)$ introduced the concepts of "generating space of $b$-dislocated quasi-metric family" (abbreviated " $G_{b d q}$-family"),"generating space of $b$-dislocated metric family" (abbreviated " $G_{b d}$-family") and "generating space of $b$-quasi-metric family" (abbreviated " $G_{b q}$-family"). Also she proved the existence of unique fixed point theorems in weaker forms of generating spaces by using various cyclic contractive conditions.

Through out this paper, we assume that $\mathbb{R}^{+}=[0, \infty), \mathbb{N}$ denotes the set of all positive integers.

Definition 1 Let $X$ be a non-empty set and $\left\{d_{\alpha}: \alpha \in(0,1]\right\}$ a family of mapping $d_{\alpha}$ of $X \times X$ in to $\mathbb{R}^{+}$. Consider the following conditions for any $x, y, z \in X$ and $s \geq 1$.

$\left(d_{1}\right) \quad$ The family of self distances are zero: $d_{\alpha}(x, x)=0$;

$\left(d_{2}\right) \quad$ The family of distances are symmetric: $d_{\alpha}(x, y)=d_{\alpha}(y, x)$;

$\left(d_{3}\right) \quad$ The family of positive distances between distinct points: $d_{\alpha}(x, y)=d_{\alpha}(y, x)=0$ implies $x=y$;

$\left(d_{4}\right) \quad$ For any $\alpha \in(0,1]$ there exist $\beta \in(0, \alpha]$ such that $d_{\alpha}(x, z) \leq s\left[d_{\beta}(x, y)+d_{\beta}(y, z)\right]$;

$\left(d_{5}\right) \quad$ For any $x, y \in X, d_{\alpha}(x, y)$ is non-increasing and left continuous in $\alpha$.

$d_{(\alpha)}$ is called,

(1) Generating space of $b$-quasi-metric family if $d_{1}$ through $d_{5}$.

(2) Generating space of $b$-dislocated metric family if $d_{2}$ through $d_{5}$.

(3) Generating space of $b$-dislocated-quasi metric family if $d_{3}$ through $d_{5}$. If $s=1$ then $G_{b q}$-family becomes generating space of quasi-metric family as defined by Banach (1922). 
Example 2 Let $(X, d)$ be a metric space. If we put $d_{\alpha}$ instead of $d$ for all $\alpha \in(0,1]$ and $x, y \in X$, then $\left(X, d_{\alpha}\right)$ is a generating space of quasi-metric family.

In Fan (1993), it was proved that each generating space of quasi-metric family generates a topology $\mathfrak{I}_{d_{\alpha}}$ whose base is the family of open balls. The " $G_{b q}$-family" will play a very predominant role in fixed point theory because the class of $G_{b q}$-family is larger than generating space of quasi-metric family.

Motivated by above, In this paper, we establish the existence of a topology induced by a Generating space of $b$-quasi-metric family. Moreover, we derive some unique fixed point theorems.

\section{Some properties of generating space of $\boldsymbol{b}$-quasi-metric family}

In 1880s, A French mathematician H Poincare introduced topological methods in studying nonlinear problems of mathematical analysis. One of main ideas was to utilize fixed point theorems. Together with the study under the topological structure derived from Poincares analysis motivation, L E J Brouwers fixed point theorem came into the world. Since then, the fixed point theory became a major branch of topology and afterwards it consistently became a major theme of the research.

Due to importance of topology in fixed point theory, we discuss some topological structures in $b$-quasi-metric family as below.

Definition 3 Let $\left(X, d_{\alpha}\right)$ be a $G_{b q}$-family and $\left\{x_{n}\right\}$ be a net in $X$. We say that $\left\{x_{n}\right\} G_{b q}$ -converges to $x$ in $\left(X, d_{\alpha}\right)$ if $\lim _{n \rightarrow \infty} d_{\alpha}\left(x_{n}, x\right)=0$ for all $\alpha \in(0,1]$.

In this case we write $\lim _{n \rightarrow \infty} x_{n}=x$.

Definition 4 Let $\left(X, d_{\alpha}\right)$ be a $G_{b q}$-family and let $A \subseteq X, x \in X$. We say that $x$ is a $G_{b q}$ -limit point of $A$ if there exists a net $\left\{x_{n}\right\}$ in $A-\left\{x_{n}\right\}$ such that $\lim _{n \rightarrow \infty} x_{n}=x$. The set of all $G_{b q^{-}}$-limit points of $A \subseteq X$ is denoted by $D(A)$.

Definition 5 Let $\left(X, d_{\alpha}\right)$ be a $G_{b q}$-family with $\epsilon>0, x \in X$. The set $\mathfrak{B}_{\epsilon}(x)=\left\{y / d_{\alpha}(x, y)<\epsilon\right\}$ is called $G_{b q}$-open ball of radius $\epsilon$ and center $x$.

The set $\overline{\mathfrak{B}_{\epsilon}}(x)=\left\{y / d_{\alpha}(x, y) \leq \epsilon\right\}$ is called $G_{b q}$-closed ball of radius $\epsilon$ and center $x$.

Remark 6 In a $G_{b q}$-family

1. $\lim _{n \rightarrow \infty} x_{n}=x$ if and only if for every $\epsilon>0$, there exists $n_{0} \in \Delta$ such that $x_{n} \in \mathfrak{B}_{\epsilon}(x)$ for all $n \geq n_{0}$.

2. $d_{\alpha}(x, y) \leq s\left[d_{\alpha}(x, z)+d_{\alpha}(z, y)\right]$ hold.

3. $G_{b q}$-limit point of a net is unique.

Now, we state some propositions and corollaries in $\left(X, d_{\alpha}, \mathfrak{I}\right)$ which can be proved following similar arguments to those given in Kumari (2012), Sarma and Kumari (2012). 
Proposition 7 Let $A, B \subseteq X$ then

1. $\mathcal{D}(A)=\phi$ if $A=\phi$

2. $\mathcal{D}(A) \subseteq \mathcal{D}(B)$ if $A \subseteq B$

3. $\mathcal{D}(A \cup B)=\mathcal{D}(A) \cup \mathcal{D}(B)$

4. $\mathcal{D}(\mathcal{D}(A)) \subseteq \mathcal{D}(A)$

Corollary 8 If we write $\bar{A}=A \cup \mathcal{D}(A)$ for $A \subset X$ the operation $A \rightarrow \bar{A}$ satisfies Kuratowski's closure axioms of Kelley (1960) so that the set $\mathfrak{I}=\left\{A / A \subset X\right.$ and $\left.\overline{A^{c}}=A^{c}\right\}$ is a topology on $X$.

Corollary 9 We call $\left(X, d_{\alpha}, \mathfrak{I}\right)$ the topological space induced by $G_{b q}$-family. We call $A \subset X$ to be closed if $\bar{A}=A$ and open if $A \in \mathfrak{I}$.

Proposition 10 Let $\left(X, d_{\alpha}\right)$ be a $G_{b q}$-family.x $\in X$ is a $G_{b q}$-limit point of $A \subset X$ iff for every $r>0, A \cap \mathfrak{B}_{r}(x) \neq \phi$.

Corollary $11 x \in \bar{A}$ if and only if $x \in A$ or $\mathfrak{B}_{r}(x) \cap A \neq \phi$, for all $r>0$.

Corollary $12 A \subseteq X$ is open in $\left(X, d_{\alpha}, \mathfrak{I}\right)$ iff for every $x \in A$, there exists $\delta>0$ such that $\mathcal{B}_{\delta}(x) \subseteq A$.

Proposition 13 If $x \in X$ and $\delta>0$, then $\mathfrak{B}_{\delta}(x)$ is an open $\operatorname{set}$ in $\left(X, d_{\alpha}, \mathfrak{I}\right)$.

Proof Let $y \in \mathfrak{B}_{\delta}(x)$ and $0<r<\frac{\delta}{s}-d_{\alpha}(x, y)$. Then $\mathfrak{B}_{r}(y) \subset \mathfrak{B}_{\delta}(x)$

Hence $\mathfrak{B}_{\delta}(x)$ is open.

Proposition $14\left(X, d_{\alpha}, \mathfrak{I}\right)$ is a Hausdorff space.

Proof Let $x \neq y$, then $d_{\alpha}(x, y)>0$. Choose $\delta>0$ such that $2 \delta<d_{\alpha}(x, y)$.

Then $\mathfrak{B}_{\frac{\delta}{s}}(x) \cap \mathfrak{B}_{\frac{\delta}{s}}(y)=\phi$.

Corollary 15 If $x \in X$, then the collection $\left\{\mathfrak{B}_{r}(x) / x \in X\right\}$ is an open base at $x$ in $\left(X, d_{\alpha}, \mathfrak{I}\right)$. Hence $\left(X, d_{\alpha}, \mathfrak{I}\right)$ is first countable.

The above corollary yields us to deal with sequences instead of nets.

Definition 16 A sequence $\left\{x_{n}\right\}$ in a $G_{b q}$-family is called a $G_{b q}$-Cauchy sequence if given $\epsilon>0$, there exists $n_{0} \in \mathbb{N}$ such that for all $n, m \geq n_{0}$, we have $d_{\alpha}\left(x_{n}, x_{m}\right)<\epsilon$ or $\lim _{n \rightarrow \infty} d_{\alpha}\left(x_{n}, x_{m}\right)=0$ for all $\alpha \in(0,1]$.

Proposition 17 Every $G_{b q}$-convergent sequence in a $G_{b q}$-family is $G_{b q}$-Cauchy.

Definition 18 A $G_{b q}$-family $\left(X, d_{\alpha}\right)$ is called complete if every $G_{b q}$-Cauchy sequence in $X$ is $G_{b q}$-Convergent. 
Remark 19 In a $G_{b q}$-family $\left(X, d_{\alpha}\right)$, a subset $A$ of $X$ is said to be closed if for any sequence $\left\{x_{n}\right\}$ of points of $A$ such that $\lim _{n \rightarrow \infty} x_{n}=x$ then $x \in A$.

\section{Main results}

Definition 20 By $\Phi$ we denote the set of all real functions $\Upsilon:[0, \infty) \rightarrow[0, \infty)$ which have the following properties:

(a) $\Upsilon$ is monotone increasing;

(b) $\quad \lim \Upsilon^{n}(t)=0$ for any $t>0$, where $\Upsilon^{n}(t)=\Upsilon \circ \cdots \circ \Upsilon(t)$.

Theorem 21 Let $\left(X, d_{\alpha}\right)$ be a complete $G_{b q}$-family with the co-efficient $s \geq 1, p>1$ and $T: X \rightarrow X$ satisfy $s^{p} d_{\alpha}(T x, T y) \leq \Upsilon\left(d_{\alpha}(x, y)\right) ; \forall x, y \in X$. where $\Phi: \mathbb{R}^{+} \rightarrow \mathbb{R}^{+}$is a continuous monotone increasing mapping such that $\lim _{n \rightarrow \infty} \Upsilon^{n}(t)=0$ for each $t>0$. Then Thas exactly one fixed point.

Proof First of all, note that, $\Upsilon(t)<t$ for all $t>0$ and $\Upsilon(0)<0$.

Let $x_{0}$ be an arbitrary point in $X$. Define the iterative sequence $\left\{x_{n}\right\}$ as follows:

$x_{1}=T\left(x_{0}\right), x_{2}=T\left(x_{1}\right), \ldots, x_{n+1}=T\left(x_{n}\right), \ldots$

If we assume that $x_{n+1}=x_{n}$ for some $n \in \mathbb{N}$, then we have $x_{n}=x_{n+1}=T\left(x_{n}\right)$, so $x_{n}$ is a fixed point of $T$ and the proof is complete. From now on we will assume that for each $n \in \mathbb{N}, x_{n+1} \neq x_{n}$.

Consider $s^{p} d_{\alpha}\left(x_{n}, x_{n+1}\right)=s^{p} d_{\alpha}\left(T x_{n-1}, T x_{n}\right) \leq \Upsilon d_{\alpha}\left(x_{n-1}, x_{n}\right)$

$d_{\alpha}\left(x_{n}, x_{n+1}\right) \leq \frac{1}{s^{p}} \Upsilon\left(d_{\alpha}\left(x_{n-1}, x_{n}\right)\right) \leq \Upsilon\left(d_{\alpha}\left(x_{n-1}, x_{n}\right)\right)$

By repeating this procedure, we get,

$$
\begin{aligned}
d_{\alpha}\left(x_{n}, x_{n+1}\right) & \leq \Upsilon d_{\alpha}\left(x_{n-1}, x_{n}\right) \\
& \leq \Upsilon^{2} d_{\alpha}\left(x_{n-2}, x_{n-1}\right) \\
& \vdots \\
& \leq \Upsilon^{n} d_{\alpha}\left(x_{0}, x_{1}\right), \quad \text { for } \mathrm{n}>1 .
\end{aligned}
$$

If $d_{\alpha}\left(x_{0}, x_{1}\right)=0$ then $x_{0}=T x_{0}$ which yields $x_{0}$ is a fixed point of $T$.

Suppose $d_{\alpha}\left(x_{0}, x_{1}\right)>0$, then $\lim _{n \rightarrow \infty} d_{\alpha}\left(x_{n}, x_{n+1}\right)=0$.

Which implies for each $\eta \geq 0$, there exists $k \in \mathbb{N}$ such that

$$
d_{\alpha}\left(T^{k}(x), T^{k+1}(x)\right) \leq \frac{\eta}{2 s} ; \forall k \geq n
$$

Now our aim is to prove $\left\{x_{n}\right\}$ is a $G_{b q}$-Cauchy sequence.

If we apply induction with respect to $n$, to show for all $n \in \mathbb{N}$,

$$
d_{\alpha}\left(T^{k}(x), T^{k+n}(x)\right) \leq \frac{\eta}{2 s} .
$$

Clearly (3) holds for $n=1$. Let us assume that (3) holds for some $n \in \mathbb{N}$.

i.e $d_{\alpha}\left(T^{k+1}(x), T^{k+n+1}(x)\right) \leq \frac{\eta}{2 s}$

We have, 


$$
\begin{aligned}
d_{\alpha}\left(T^{k}(x), T^{k+n}(x)\right) & \leq \frac{1}{s^{p}} \Upsilon\left(d_{\alpha}\left(T^{k}(x), T^{k+n}(x)\right)\right) \\
& \leq \frac{1}{s^{p}} \Upsilon\left(\frac{\eta}{2 s}\right) \\
& <\frac{\eta}{2 s} .
\end{aligned}
$$

Now consider,

$$
\begin{aligned}
d_{\alpha}\left(T^{k}(x), T^{k+n+1}(x)\right) & \leq s\left[d_{\alpha}\left(T^{k}(x), T^{k+1}(x)\right)+d_{\alpha}\left(T^{k+1}(x), T^{k+n+1}(x)\right)\right] \\
& \leq s\left[\frac{\eta}{2 s}+\frac{\eta}{2 s}\right] \\
& <\eta .
\end{aligned}
$$

Thus by induction, we get, (3) is satisfied for any $n \geq 1$.

Hence $d_{\alpha}\left(T^{k}(x), T^{k+n}(x)\right)<\eta$. which yields that $\left\{x_{n}\right\}$ is a $G_{b q}$-Cauchy sequence in a complete $G_{b q}$-family. Thus there exists some $u$ in $X$ such that $\lim _{n \rightarrow \infty} x_{n}=u$. Also the subsequence $\left\{x_{n+1}\right\} G_{b q}$-converges to $u$ in $X$.

For any $x, y$ in $X$, we have

$$
\begin{aligned}
d_{\alpha}(T x, T y) & \leq \frac{1}{s^{p}} \Upsilon\left(d_{\alpha}(x, y)\right) \\
& <\Upsilon\left(d_{\alpha}(x, y)\right) \\
& \leq d_{\alpha}(x, y) .
\end{aligned}
$$

which implies $T$ is continuous. Hence $\lim _{n \rightarrow \infty} T x_{n}=T u$.

Consider $d_{\alpha}(u, T u) \leq s\left[d_{\alpha}\left(u, x_{n+1}\right)+d_{\alpha}\left(x_{n+1}, T u\right)\right]$.

By taking limits $n \rightarrow \infty, d_{\alpha}(u, T u)=0$.

Hence $u=T u$.

Uniqueness: Let $u, v$ be two fixed points of $T$ and $u \neq v$.

Thus $d_{\alpha}(u, v) \neq 0$ which implies $d_{\alpha}(u, v)>0$.

Consider,

$$
\begin{aligned}
s^{p} d_{\alpha}(u, v) & =s^{p} d_{\alpha}(T u, T v) \\
& \leq \Upsilon d_{\alpha}(u, v) \\
& <d_{\alpha}(u, v) .
\end{aligned}
$$

A contradiction. Thus $d_{\alpha}(u, v)=0$. Which implies $u=v$.

Hence $T$ has a unique fixed point.

This completes the proof of the theorem.

By taking $\Upsilon(t)=\lambda t$ with $0 \leq \lambda<\frac{1}{s^{p}}$, we can set the following corollary which generalizes the famous Banach contraction principle in $G_{b q}$-family.

Corollary 22 Let $\left(X, d_{\alpha}\right)$ be a complete $G_{b q}$-family with the coefficient $s \geq 1, p>1$ and let $T: X \rightarrow X$ is a mapping such that $d_{\alpha}(T x, T y) \leq \lambda d_{\alpha}(x, y)$ for all $x, y \in X$, where $0 \leq \lambda<\frac{1}{s^{p}}$. Then $T$ has a unique fixed point in $X$.

By taking $s=1$ in above corollary, we generalize the Banach contraction principle in generating space of quasi-metric family. 
Corollary 23 Let $\left(X, d_{\alpha}\right)$ be a complete generating space of quasi-metric family with the coefficient $s>1$, and let $T: X \rightarrow X$ be a mapping such that $d_{\alpha}(T x, T y) \leq \lambda d_{\alpha}(x, y)$ for all $x, y \in X$, where $0 \leq \lambda<1$. Then Thas a unique fixed point in $X$.

By taking $d_{\alpha}=d$ in above corollary, we get Banach contraction principle in complete metric space.

Rhoades (1977) collected some contractive conditions considered by various authors and established implications and non-implications between them. We noted some contractive conditions as mentioned below.

Let $(X, d)$ be a metric space. If $T: X \rightarrow X$ is a self mapping and $x, y$ be any elements of $X$. Now consider the following contractive conditions:

(Rhoades 1977) There exist nonnegative functions $a, b$, c satisfying

$$
\sup _{x, y \in X}\{a(x, y)+b(x, y)+c(x, y)\} \leq \lambda<1
$$

such that, for each $x, y \in X$,

$$
d(T x, T y) \leq a(x, y) d(x, T y)+b(x, y) d(y, T x)+c(x, y) d(x, y) .
$$

Ciric.1 (Ciric 1974) There exists nonnegative functions $q, r, s, t$ satisfying

$$
\sup _{x, y \in X}\{q(x, y)+r(x, y)+s(x, y)+2 t(x, y)\} \leq \lambda<1
$$

such that, for each $x, y \in X$,

$d(T x, T y) \leq q(x, y) d(x, y)+r(x, y) d(x, T x)+s(x, y) d(y, T y)+t(x, y)[d(x, T y)+d(y, T x)]$.

Ciric.2 (Ciric 1971) There exist a constant $h, 0 \leq h<1$, such that for each $x, y \in X$,

$$
d(T x, T y) \leq h . m a x\{d(x, y), d(x, T x), d(y, T y), d(x, T y), d(y, T x)\}
$$

Note that above mentioned named contractions, as originally defined by their respective authors. In above contractions, Ciric. 2 condition is very significant because, a good number of contractive conditions imply Ciric. 2 condition.

Based on the definition of quasi-contraction of Ciric (1971), we introduce the following definition in the setting of $G_{b q}$-family.

Definition 24 Let $\left(X, d_{\alpha}\right)$ be a complete $G_{b q}$-family with the parameter $s \geq 1, p>1$. If $T: X \rightarrow X$ be a self mapping which satisfies

$$
s^{p} d_{\alpha}(T x, T y) \leq h \cdot \max \left\{d_{\alpha}(x, y), d_{\alpha}(x, T x), d_{\alpha}(y, T y), d_{\alpha}(x, T y), d_{\alpha}(y, T x)\right\}
$$

for all $x, y \in X$ and $h \in\left[0, \frac{s^{p-1}}{s+1}\right)$. Then $T$ is called "s-h generating $b$-quasi-contraction".

Definition 25 Let $\left(X, d_{\alpha}\right)$ be a complete $G_{b q}$-family with the parameter $s \geq 1, p>1$. If $T: X \rightarrow X$ is a self continuous mapping which satisfies $s$ - $h$ generating $b$-quasi-contraction, then $T$ has a unique fixed point in $X$.

Proof Let $x_{0}$ be an arbitrary point in $X$. Define the iterative sequence $\left\{x_{n}\right\}$ as follows: 


$$
x_{1}=T\left(x_{0}\right), x_{2}=T\left(x_{1}\right), \ldots, x_{n+1}=T\left(x_{n}\right), \ldots
$$

If we assume that $x_{n+1}=x_{n}$ for some $n \in \mathbb{N}$, then we have $x_{n}=x_{n+1}=T\left(x_{n}\right)$, so $x_{n}$ is a fixed point of $T$ and the proof is complete. Now we will assume that for each $n \in \mathbb{N}, x_{n+1} \neq x_{n}$.

Now consider,

$$
\begin{aligned}
s^{p} d_{\alpha}\left(x_{n}, x_{n+1}\right) & =s^{p} d_{\alpha}\left(T x_{n-1}, T x_{n}\right) \\
& \leq h \cdot m a x\left\{d_{\alpha}\left(x_{n-1}, x_{n}\right), d_{\alpha}\left(x_{n-1}, x_{n}\right), d_{\alpha}\left(x_{n}, x_{n+1}\right), d_{\alpha}\left(x_{n-1}, x_{n+1}\right), d_{\alpha}\left(x_{n}, x_{n}\right)\right\} \\
& \leq h \cdot m a x\left\{d_{\alpha}\left(x_{n-1}, x_{n}\right), d_{\alpha}\left(x_{n}, x_{n+1}\right), s\left[d_{\alpha}\left(x_{n-1}, x_{n}\right)+d_{\alpha}\left(x_{n}, x_{n+1}\right)\right]\right\}
\end{aligned}
$$

which implies that,

$$
\begin{aligned}
d_{\alpha}\left(x_{n}, x_{n+1}\right) & \leq \frac{h s}{s^{p}-h s} d_{\alpha}\left(x_{n-1}, x_{n}\right) \\
& \leq \frac{h}{s^{p-1}-h} d_{\alpha}\left(x_{n-1}, x_{n}\right) \\
& \leq c \cdot d_{\alpha}\left(x_{n-1}, x_{n}\right) .
\end{aligned}
$$

where $c=\frac{h}{s^{p-1}-h}$.

Similarly, by the contractive condition of the theorem, we can get below condition:

$$
d_{\alpha}\left(x_{n-1}, x_{n}\right) \leq c . d_{\alpha}\left(x_{n-2}, x_{n-1}\right)
$$

By repeating the same process, we get for all $n \geq 2$.

$$
\begin{aligned}
d_{\alpha}\left(x_{n}, x_{n+1}\right) & \leq c . d_{\alpha}\left(x_{n-1}, x_{n}\right) \\
& \leq \\
& \vdots \\
& \leq c^{n} \cdot d_{\alpha}\left(x_{0}, x_{1}\right)
\end{aligned}
$$

Since $0 \leq c<1$ and applying limits as $n \rightarrow \infty$, we get $d_{\alpha}\left(x_{n}, x_{n+1}\right) \rightarrow 0$.

Now our aim is to prove $\left\{x_{n}\right\}$ is a $G_{b q}$-Cauchy sequence.

To obtain this, let $m, n>0$ with $m>n$.

Then we have,

$$
\begin{aligned}
d_{\alpha}\left(x_{n}, x_{m}\right) & \leq s\left[d_{\alpha}\left(x_{n}, x_{n+1}\right)+d_{\alpha}\left(x_{n+1}, x_{m}\right)\right] \\
& \leq s d_{\alpha}\left(x_{n}, x_{n+1}\right)+s^{2} d_{\alpha}\left(x_{n+1}, x_{n+2}\right)+s^{3} d_{\alpha}\left(x_{n+2}, x_{n+3}\right)+\cdots \\
& \leq s c^{n} d_{\alpha}\left(x_{0}, x_{1}\right)+s^{2} c^{n+1} d_{\alpha}\left(x_{0}, x_{1}\right)+s^{3} c^{n+2} d_{\alpha}\left(x_{0}, x_{1}\right)+\cdots \\
& =s c^{n} d_{\alpha}\left(x_{0}, x_{1}\right)\left[1+s c+(s c)^{2}+(s c)^{3}+\cdots\right] \\
& \leq \frac{s c^{n}}{1-s c} d_{\alpha}\left(x_{0}, x_{1}\right) .
\end{aligned}
$$

By taking the limits as $n, m \rightarrow \infty$, we get $d_{\alpha}\left(x_{n}, x_{m}\right) \rightarrow 0$ as $c s<1$.

Hence $\left\{x_{n}\right\}$ is a $G_{b q}$-Cauchy sequence in complete $G_{b q}$-family $\left(X, d_{\alpha}\right)$. Thus there exists some $u \in X$ such that $\left\{x_{n}\right\} G_{b q}$-converges to $u$.

Since $T$ is a continuous mapping,

$$
T(u)=T\left(\lim _{n \rightarrow \infty} x_{n}\right)=\lim _{n \rightarrow \infty} T\left(x_{n}\right)=\lim _{n \rightarrow \infty}\left(x_{n+1}\right)=u .
$$


Hence $u$ is a fixed point of $T$.

Uniqueness Let us suppose that $u$ and $v$ are two fixed points of $T$ where $T u=u$ and $T v=v$. Then by s-h generating b-quasi-contraction, we get

$$
\begin{aligned}
s^{p} d_{\alpha}(u, v) & =s^{p}\left[d_{\alpha}(T u, T v)\right] \\
& \leq h \cdot \max \left\{d_{\alpha}(u, v), d_{\alpha}(u, T u), d_{\alpha}(v, T v), d_{\alpha}(u, T v), d_{\alpha}(v, T u)\right\} \\
& =h \cdot \max \left\{d_{\alpha}(u, v), d_{\alpha}(u, u), d_{\alpha}(v, v), d_{\alpha}(u, v), d_{\alpha}(v, u)\right\} \\
& \leq h d_{\alpha}(u, v)
\end{aligned}
$$

So $d_{\alpha}(u, v) \leq k \cdot d_{\alpha}(u, v)$ where $k=\frac{h}{s^{p}}$ and since $0 \leq k<1$, then we get $d_{\alpha}(u, v)=0$.

Hence $d_{\alpha}(u, v)=0$ which implies $u=v$.

Hence $T$ has a unique fixed point in $X$.

If we take parameter $s=1$ in the above theorem, we obtain following corollary.

Corollary 26 Let $\left(X, d_{\alpha}\right)$ be a complete generating space of quasi-metric family and $T: X \rightarrow X$ is a self continuous mapping which satisfies:

$$
d_{\alpha}(T x, T y) \leq h . m a x\left\{d_{\alpha}(x, y), d_{\alpha}(x, T x), d_{\alpha}(y, T y), d_{\alpha}(x, T y), d_{\alpha}(y, T x)\right\}
$$

for all $x, y \in X$ and $h \in\left[0, \frac{1}{2}\right)$. Then Thas a unique fixed point in $X$.

If we put $d_{\alpha}=d$ in above corollary, we get following corollary.

Corollary 27 Let $(X, d)$ be a complete metric space and if $T: X \rightarrow X$ is a self continuous mapping which satisfies:

$$
d(T x, T y) \leq h \cdot m a x\{d(x, y), d(x, T x), d(y, T y), d(x, T y), d(y, T x)\}
$$

for all $x, y \in X$ and $h \in\left[0, \frac{1}{2}\right)$. Then Thas a unique fixed point in $X$.

We now give an example to illustrate the above corollary.

Example 28 Let $X=[0,1]$ and $d(x, y)=|x-y| ; \forall x, y \in X$.

Clearly $d$ is a complete metric on $X$. Define the self mapping $T: X \rightarrow X$ by $T(x)=\frac{x}{3}$.

For $x, y \in[0,1]$, we have

$$
\begin{aligned}
d(T x, T y) & =\left|\frac{x-y}{3}\right| \\
& =\frac{1}{3}|x-y| \\
& =\frac{1}{3} d(x, y) \\
& \leq h \cdot \max \{d(x, y), d(x, T x), d(y, T y), d(x, T y), d(y, T x)\}
\end{aligned}
$$

for $\frac{1}{3} \leq h<\frac{1}{2}$. Clearly $x=0$ is the unique fixed point of $T$.

Three eminent conditions (1), (2) and (3) are made significant contribution in the area of fixed point theory and applications. After these three results, a huge number of papers have been written by several authors to those results either improve or generalize some of the conditions (1), (2) or (3), or even the three conditions simultaneously. 
In 1972, Zamfirescu (1972) consolidate the (1,2,3) conditions which is known as Zamfirescu contractive condition and proved a fixed point theorem.

(Zamfirescu 1972) There exists real numbers $\eta, \beta, \gamma, 0 \leq \eta<1,0 \leq \beta, \gamma<\frac{1}{2}$, such that for each $x, y \in X$ at least one of the following is true:

1. $d(T x, y) \leq \eta d(x, y)$,

2. $d(T x, T y) \leq \beta[d(x, T x)+d(y, T y)]$,

3. $d(T x, T y) \leq \gamma[d(x, T y)+d(y, T x)]$.

Then $T$ has a fixed point.

In Rhoades (1977), Rhoades state below conditions,

(Rhoades 19' Rhoades 1977) There exist non-negative functions $a, b, c$ satisfying $\sup _{x, y \in X}\{a(x, y)+2 b(x, y)+2 c(x, y)\} \leq \lambda<1$ such that, for each $x, y \in X$,

$$
\begin{aligned}
d(f(x), f(y)) \leq & a(x, y) d(x, y)+b(x, y)[d(x, f(x))+d(y, f(y))] \\
& +c(x, y)[d(x, f(y))+d(y, f(x))]
\end{aligned}
$$

(Rhoades 19" Rhoades 1977) There exist a constant $h, 0 \leq h<1$, such that for each $x, y \in X$,

$d(f(x), f(y)) \leq h \max \left\{d(x, y), \frac{[d(x, f(x))+d(y, f(y))]}{2}, \frac{[d(x, f(y))+d(y, f(x))]}{2}\right\}$

Moreover, Rhoades proved that Zamfirescus condition is equivalent to Rhoades $19^{\prime}$ \&Rhoades 19" conditions. However, recently Berinde (2004) proved that Banach's, Kannan's, Chatterjea's and Zamfirescu's mappings are weak contractions.

Theorem 29 Let $(X, d)$ is a complete metric space. If $T: X \rightarrow X$ be a self continuous mapping satisfying any of the conditions either Rhodes or Zamfirescu or Ciric.1. Then T has a unique fixed point.

Proof In Rhoades (1977), Rhodes proved below Implications.

$$
\begin{aligned}
& \text { Rhodes } \Rightarrow \text { Ciric. } 2 \\
& \text { Zamfirescu } \Rightarrow \text { Ciric. } 1 \Rightarrow \text { Ciric. } 2
\end{aligned}
$$

Hence from Corollary 27, $T$ has a unique fixed point.

Theorem 30 Let $\left(X, d_{\alpha}\right)$ be a complete $G_{b q}$-family with the parameters $s \geq 1$ and $p \geq 1$. If $T: X \rightarrow X$ is a self continuous mapping which satisfies $s$ - $h$ generating $b$-quasi-contraction, i.e.

$$
s^{p} d_{\alpha}(T x, T y) \leq h \cdot \max \left\{d_{\alpha}(x, y), d_{\alpha}(x, T x), d_{\alpha}(y, T y), d_{\alpha}(x, T y), d_{\alpha}(y, T x)\right\}
$$

for all $x, y \in X$ and $h \in\left[0, \frac{s^{p-1}}{s+1}\right)$. If for some positive integer $q, T^{q}$ is continuous, then $T$ has a unique fixed point in $X$. 
Proof We can construct a sequence $\left\{x_{n}\right\}$ as in Theorem 30 and conclude that the sequence $\left\{x_{n}\right\} G_{b q}$-converges to some point $u$ in $X$.

Thus its subsequence $\left\{x_{n_{k}}\right\}\left(n_{k}=k\right) G_{b q}$-converges to $u$. Also we have,

$T^{q}(u)=T^{q}\left(\lim _{k \rightarrow \infty} x_{n_{k}}\right)=\lim _{k \rightarrow \infty}\left(T^{q}\left(x_{n_{k}}\right)\right)=\lim _{k \rightarrow \infty}\left(x_{k+q}\right)=u$

Which yields that $u$ is a fixed point of $T^{q}$.

Now we shall prove that $T u=u$.

Let $l$ be a smallest positive integer such that $T^{l} u=u$ but $T^{m} u \neq u \cdot(m=1,2, \ldots l-1)$. If $l>1$ then,

$$
\begin{aligned}
s^{p} d_{\alpha}(u, T u) & =s^{p} d_{\alpha}\left(T^{l} u, T u\right) \\
& =s^{p} d_{\alpha}\left(T T^{l-1} u, T u\right) \\
& \leq h \cdot \max \left\{d_{\alpha}\left(T^{l-1} u, u\right), d_{\alpha}\left(T^{l-1} u, T^{l} u\right), d_{\alpha}(u, T u), d_{\alpha}\left(T^{l-1} u, T u\right), d_{\alpha}\left(u, T^{l} u\right)\right\} \\
& \leq h \cdot \max \left\{d_{\alpha}\left(T^{l-1} u, u\right), d_{\alpha}(u, T u), s\left[d_{\alpha}\left(T^{l-1} u, u\right)+d_{\alpha}(u, T u)\right]\right\} \\
& \leq h s d_{\alpha}\left(T^{l-1} u, u\right)+h s d_{\alpha}(u, T u)
\end{aligned}
$$

which yields,

$$
\begin{aligned}
d_{\alpha}(u, T u) & \leq \frac{h s}{s^{p}-h s} d_{\alpha}\left(T^{l-1} u, u\right) \\
& \leq \frac{h}{s^{p-1}-h} d_{\alpha}\left(T^{l-1} u, u\right) .
\end{aligned}
$$

Thus $d_{\alpha}(u, T u)<k \cdot d_{\alpha}\left(T^{l-1} u, u\right)$; where $k=\frac{h}{s^{p-1}-h}$.

Similarly,

$$
\begin{aligned}
s^{p} d_{\alpha}\left(T^{l-1} u, u\right)= & s^{p} d_{\alpha}\left(T T^{l-2} u, T T^{l-1} u\right) \\
\leq & h \cdot \max \left\{d_{\alpha}\left(T^{l-2} u, T^{l-1} u\right), d_{\alpha}\left(T^{l-2} u, T^{l-1} u\right), d_{\alpha}\left(T^{l-1} u, T^{l} u\right)\right. \\
& \left.d_{\alpha}\left(T^{l-2} u, T^{l} u\right), d_{\alpha}\left(T^{l-1} u, T^{l-1} u\right)\right\} \\
\leq & h \cdot \max \left\{d_{\alpha}\left(T^{l-2} u, T^{l-1} u\right), d_{\alpha}\left(T^{l-1} u, u\right), s\left[d_{\alpha}\left(T^{l-2} u, T^{l-1} u\right)+d_{\alpha}\left(T^{l-1} u, u\right)\right]\right\} \\
\leq & h s\left[d_{\alpha}\left(T^{l-2} u, T^{l-1} u\right)+d_{\alpha}\left(T^{l-1} u, u\right)\right]
\end{aligned}
$$

Which implies,

$$
\begin{aligned}
d_{\alpha}\left(T^{l-1} u, u\right) & \leq \frac{h s}{s^{p}-h s} d_{\alpha}\left(T^{l-2} u, T^{l-1} u\right) \\
& \leq \frac{h}{s^{p-1}-h} d_{\alpha}\left(T^{l-2} u, T^{l-1} u\right)
\end{aligned}
$$

Inductively we get,

$$
\begin{aligned}
d_{\alpha}\left(T^{l-1} u, u\right) & =d_{\alpha}\left(T^{l-1} u, T^{l} u\right) \\
& \leq k \cdot d_{\alpha}\left(T^{l-2} u, T^{l-1} u\right) \\
& \vdots \\
& \leq k^{l-1} \cdot d_{\alpha}(u, T u)
\end{aligned}
$$

notice that $k<1$. 
Thus $d_{\alpha}(u, T u) \leq k^{l-1} . d_{\alpha}(u, T u) \leq d_{\alpha}(u, T u)$

Which is a contradiction.

Hence $T u=u$.

Which yields $u$ is a fixed point of $T$.

Now if there exists another point $v \neq u$ in $X$ such that $T v=v$, then

$$
\begin{aligned}
& s^{p} d_{\alpha}(u, v)=s^{p} d_{\alpha}(T u, T v) \\
& \leq h \cdot \max \left\{d_{\alpha}(u, v), d_{\alpha}(u, T u), d_{\alpha}(v, T v), d_{\alpha}(u, T v), d_{\alpha}(v, T u)\right\} \\
& \leq h \cdot \max \left\{d_{\alpha}(u, v), d_{\alpha}(u, u), d_{\alpha}(v, v), d_{\alpha}(u, v), d_{\alpha}(v, u)\right\} \\
& \leq h d_{\alpha}(u, v)
\end{aligned}
$$

Which implies $d_{\alpha}(u, v) \leq \frac{h}{s^{p}} d_{\alpha}(u, v)<d_{\alpha}(u, v)$. A contradiction.

Hence $u$ is a unique fixed point of $T$ in $X$.

If we take parameter $s=1$ in the above theorem, we obtain following corollary in generating spaces of quasi-metric family.

Corollary 31 Let $\left(X, d_{\alpha}\right)$ be a complete generating space of quasi-metric family and $T: X \rightarrow X$ is a continuous mapping which satisfies:

$$
d_{\alpha}(T x, T y) \leq h \cdot m a x\left\{d_{\alpha}(x, y), d_{\alpha}(x, T x), d_{\alpha}(y, T y), d_{\alpha}(x, T y), d_{\alpha}(y, T x)\right\}
$$

for all $x, y \in X$ and $h \in\left[0, \frac{1}{2}\right)$.Iffor some positive integer $q, T^{q}$ is continuous, then $T$ has a unique fixed point in $X$.

If we take $d_{\alpha}=d$ then we get following corollary.

Corollary 32 Let $(X, d)$ be a complete metric space and if $T: X \rightarrow X$ is a continuous mapping which satisfies:

$$
d(T x, T y) \leq h \cdot \max \{d(x, y), d(x, T x), d(y, T y), d(x, T y), d(y, T x)\}
$$

for all $x, y \in X$ and $h \in\left[0, \frac{1}{2}\right)$.Iffor some positive integer $q, T^{q}$ is continuous, then $T$ has a unique fixed point in $X$.

Based on the definition of Zamfirescu Contraction, we introduce the following definition in the setting of $G_{b q}$-family.

Definition 33 Let $\left(X, d_{\alpha}\right)$ be a complete $G_{b q}$-family with the parameter $s \geq 1, p \geq 1$. If $f: X \rightarrow X$ be a mapping such that for each $x, y \in X$ at least one of the following is true:

1. $s^{p} d_{\alpha}(f(x), f(y)) \leq \eta d_{\alpha}(x, y), 0 \leq \eta<s^{p-1}$

2. $s^{p} d_{\alpha}(f(x), f(y)) \leq \beta\left[d_{\alpha}(x, f(x))+d_{\alpha}(y, f(y))\right], 0 \leq \beta<\frac{s^{p}}{s+1}$

3. $s^{p} d_{\alpha}(f(x), f(y)) \leq \gamma\left[d_{\alpha}(x, f(y))+d_{\alpha}(y, f(x))\right], 0 \leq \gamma<\frac{s^{p-1}}{s+1}$

Then $f$ is called "s-z generating b-quasi-contraction". 
Theorem 34 Let $\left(X, d_{\alpha}\right)$ be a complete $G_{b q}$-family with the parameters $s>1, p>1$. If $f: X \rightarrow X$ be a continuous $s$ - $z$ generating $b$-quasi-contraction then $f$ has a unique fixed point in $X$.

Proof Put $y=f(x)$ in the above (1), (2) and (3) of Definition 33, gives,

$$
\begin{array}{r}
d_{\alpha}\left(f(x), f^{2}(x)\right) \leq \frac{\eta}{s^{p}} d_{\alpha}(x, f(x)) \\
d_{\alpha}\left(f(x), f^{2}(x)\right) \leq \frac{\beta}{s^{p}-\beta} d_{\alpha}(x, f(x)) \\
d_{\alpha}\left(f(x), f^{2}(x)\right) \leq \frac{\gamma s}{s^{p}-\gamma s} d_{\alpha}(x, f(x))
\end{array}
$$

Choose $h=\max \left\{\frac{\eta}{s^{p}}, \frac{\beta}{s^{p}-\beta}, \frac{\gamma s}{s^{p}-\gamma s}\right\}$ and $0 \leq h<\frac{1}{s}$ then we get

$$
d_{\alpha}\left(f(x), f^{2}(x)\right) \leq h d_{\alpha}(x, f(x))
$$

By repeating this procedure,we obtain

$$
d_{\alpha}\left(f^{n}(x), f^{n+1}(x)\right) \leq h^{n} d_{\alpha}(x, f(x))
$$

Since $0 \leq h<\frac{1}{s}, \lim _{n \rightarrow \infty} d_{\alpha}\left(f^{n}(x), f^{n+1}(x)\right) \rightarrow 0$.

Now we prove that $f^{n}(x)$ is a $G_{b q}$-Cauchy sequence.

To do this,let $m, n$ are positive integers such that $m>n$.

Now consider

$$
\begin{aligned}
d_{\alpha}\left(f^{n}(x), f^{m}(x)\right) & \leq s\left[d_{\alpha}\left(f^{n}(x), f^{n+1}(x)\right)+d_{\alpha}\left(f^{n+1}(x), f^{m}(x)\right)\right] \\
& \leq s d_{\alpha}\left(f^{n}(x), f^{n+1}(x)\right)+s^{2} d_{\alpha}\left(f^{n+1}(x), f^{n+2}(x)\right)+s^{3} d_{\alpha}\left(f^{n+2}(x), f^{n+3}(x)\right)+\cdots \\
& \leq s^{n} d_{\alpha}(x, f(x))+s^{2} h^{n+1} d_{\alpha}(x, f(x))+\cdots \\
& \leq \operatorname{sh}^{n}\left[1+s h+(s h)^{2}+\cdots\right] d_{\alpha}(x, f(x)) \\
& \leq \frac{s^{n}}{1-s h} d_{\alpha}(x, f(x))
\end{aligned}
$$

Applying $\lim _{n, m \rightarrow \infty}$, we get, $d_{\alpha}\left(f^{n}(x), f^{m}(x)\right) \rightarrow 0$ as $h s<1$.

Thus $f^{n}(x)$ is a $G_{b q}$-Cauchy sequence in complete $G_{b q}$-family.

Which implies there exist some $u \in X$ such that $\lim _{n \rightarrow \infty} d_{\alpha}\left(f^{n}(x), u\right)=0$.

Since $f$ is continuous, we get $f(u)=f\left(\lim _{n \rightarrow \infty}\left(f^{n}(x)\right)=\lim _{n \rightarrow \infty} f^{n+1}(x)=u\right.$.

Thus $u$ is a fixed point of $f$. Suppose there exists another point $v \neq u$ in $X$ such that $f(v)=v$ then $d_{\alpha}(u, v)=d_{\alpha}(f(u), f(v)) \leq h d_{\alpha}(u, v)$.

Since $0 \leq h<1, d_{\alpha}(u, v)=0$. Which implies $u=v$. Hence $u$ is a unique fixed point.

We now give an example to illustrate the above theorem.

Example 35 Let $X=[0,1]$. Define $d_{\alpha}: X \times X \rightarrow \mathbb{R}^{+}$by $d_{\alpha}(x, y)=|x-y|$ for all $\alpha \in(0,1]$ then $d_{\alpha}$ is a complete $G_{b q}$-family. Let $f: X \rightarrow X$ be a mapping defined by $f x=\frac{x}{2}$ then for any $x, y \in X$.

$$
\frac{|x-y|}{2}<|x-y|<\frac{\eta}{s^{p}}|x-y|
$$


which implies, $s^{p \frac{|x-y|}{2}<\eta|x-y|}$

$$
\text { i.e. } s^{p} d_{\alpha}(f(x), f(y))<\eta d_{\alpha}(x, y)
$$

Hence $f$ satisfies the condition(1) of Theorem 34 but $f$ doesn't satisfy condition(2) of Theorem 34 .

Since

$$
s^{p} d_{\alpha}(f(0), f(1)) \not \leq \beta\left[d_{\alpha}(0, f(0))+d_{\alpha}(1, f(1))\right] .
$$

Hence $x=0$ is the unique fixed point of $f$ in $X$.

If we take parameter $s=1$ in the above theorem, we obtain following corollary in generating spaces of quasi-metric family.

Corollary 36 Let $\left(X, d_{\alpha}\right)$ be a complete generating spaces of quasi-metric family. If $f: X \times X \rightarrow \mathbb{R}^{+}$be a continuous mapping such that for each $x, y \in X$ at least one of the following is true.

1. $d_{\alpha}(f(x), f(y)) \leq \eta d_{\alpha}(x, y), 0 \leq \eta<1$

2. $d_{\alpha}(f(x), f(y)) \leq \beta\left[d_{\alpha}(x, f(x))+d_{\alpha}(y, f(y))\right], 0 \leq \beta<\frac{1}{2}$

3. $d_{\alpha}(f(x), f(y)) \leq \gamma\left[d_{\alpha}(x, f(y))+d_{\alpha}(y, f(x))\right], 0 \leq \gamma<\frac{1}{2}$ Then $f$ has a unique fixed point.

If we take $d_{\alpha}=d$ then we get following corollary.

Corollary 37 Let $(X, d)$ be a complete metric space. If $f: X \times X \rightarrow \mathbb{R}^{+}$be a continuous mapping such that for each $x, y \in X$ at least one of the following is true.

1. $d(f(x), f(y)) \leq \eta d(x, y), 0 \leq \eta<1$

2. $d(f(x), f(y)) \leq \beta[d(x, f(x))+d(y, f(y))], 0 \leq \beta<\frac{1}{2}$

3. $d(f(x), f(y)) \leq \gamma[d(x, f(y))+d(y, f(x))], 0 \leq \gamma<\frac{1}{2}$ Then $f$ has a unique fixed point.

Open Question What are the additional conditions as needed in order to establish the existence of a unique fixed point satisfying the condition for any $\alpha \in(0,1]$ there exists $\beta \in(0, \alpha)$ such that $d_{\alpha}(x, z) \leq s\left[d_{\beta}(x, y)+d_{\beta}(y, z)\right]$ in $G_{b q}$-family $\left(X, d_{\alpha}\right)$.

Conclusion In this work, we introduced a new concept of $s$ - $h$ generating $b$-quasi-contraction., $s-z$ generating b-quasi-contraction. Also, we derived the existence of fixed point theorems for generating spaces of b-quasi-metric family. Moreover, some examples are provided wherever necessary. Our results may be the motivation to other authors for extending and improving these results to be suitable tools for their applications.

Authors' contributions

PSK and MS contributed equally to the writing of this paper. Both authors read and approved the final manuscript.

Author details

${ }^{1}$ Department of Mathematics, National Institute of Technology-A.P, Tadepalligudem, Andhra Pradesh, India. ${ }^{2}$ Department of Mathematics, University of Malakand, Chakdara Dir(L), Khyber PakhtunKhwa, Pakistan.

\section{Acknowledgements}

The first author would like to express her sincere gratitude to Mr. Anand Prabhakar for his invaluable support and motivation. The authors would like to express their thanks to the referees for their helpful comments and suggestions. The authors are grateful to Springerplus for granting full fee waiver. 


\section{Competing interests}

The authors declare that they have no competing interests.

Received: 18 August 2015 Accepted: 16 February 2016

Published online: 03 March 2016

\section{References}

Bakhtin IA (1989) The contraction mapping principle in quasi-metric spaces. Funct Anal (Ulyanovsk) 30:26-37

Banach S (1922) Sur les operations dans les ensembles abstraits et leur applications aux equations integrales. Fund Math 3:133-181

Berinde V (2004) Approximating fixed points of weak contractions using the Picard iteration. Nonlinear Anal Forum 9:43-53

Branciari A (2000) A fixed point theorem of Banach-Caccioppoli type on a class of generalized metric spaces. Publ Math (Debr) 57:31-37

Chang SS, Cho YJ, Lee BS, Jung JS, Kang SM (1997) Coincidence point theorems and minimization theorems in fuzzy metric spaces. Fuzzy Sets Syst 88:119-127

Chatterjea SK (1972) Fixed-point theorems. C R Acad Bulgare Sci 25:727-730

Ciric LB (1974) A generalization of Banach's contraction principle. Proc Am Math Soc 45:267-273 MR 50 \#8484

Ciric LB (1971) Generalized contractions and fixed-point theorems. Publ Inst Math (Beograd) (NS) 12(26):19-26 MR 46 \#8203

Fan JX (1993) On the generalizations of Ekeland's variational principle and Caristi's fixed point theorem. In: The 6th National conference on the fixed point theory, variational inequalities and probabilistic metric spaces theory, Qingdao, China

Kannan R (1968) Some results on fixed points. Bull Calcutta Math Soc 60:71-76

Kelley JL (1960) General topology. D. Van Nostrand Company, Inc, New York

Kumari PS (2012) On dislocated quasi metrics. J Adv Stud Topol 3(2):66-74

Kumari Panda Sumati, Kumar Vemuri Vasantha, Sarma Ivaturi Rambhadra (2012) Common fixed point theorems on weakly compatible maps on dislocated metric spaces. Math Sci 6:71

Kumari Panda Sumati, Panthi Dinesh (2016) Connecting various types of cyclic contractions and contractive self-mappings with Hardy-Rogers self-mappings. Fixed Point Theory Appl 2016(1):1-19

Kumari Panda S, Panthi Dinesh (2015) Cyclic contractions and fixed point theorems on various generating spaces. Fixed Point Theory Appl 2015(1):153

Kumari PSumati, Ramana Ch V (2014) On quasi-symmetric space. Indian J Sci Technol 7(10):1583-1587

Kumari PS, Sarma IR, Rao JM (2015) Metrization theorem for a weaker class of uniformities. Afr Math. doi:10.1007/ s13370-015-0369-9

Kumari PSumati, Zoto Kastriot, Panthi Dinesh (2015) d-Neighborhood system and generalized F-contraction in dislocated metric space. SpringerPlus 4(1):1-10

Lee GM, Lee BS, Jung JS, Chang SS (1999) Minimization theorems and fixed point theorems in generating spaces of quasi-metric family. Fuzzy Sets Syst 101:143-152

Matthews SG (1986) Metric domains for completeness. Technical Report 76 [Ph.D. thesis], Department of Computer Science, University of Warwick, Coventry, UK

Rhoades BE (1977) A comparison of various definitions of contractive mappings. Trans Am Math Soc 226:257-290

Ramabhadra Sarma I, Madhusudana Rao J, Sumati Kumari P, Panthi D (2014) Convergence axioms on dislocated symmetric spaces. Abst Appl Anal. doi:10.1155/2014/745031

Sarma IR, Kumari PS (2012) On dislocated metric spaces. Int J Math Achieve 3(1):7-27

Subrahmanyam PV (1975) Completeness and fixed-points. Monatshefte Math 80:325-330

Zamfirescu T (1972) Fixed point theorems in metric spaces. Arch Math 23:292-298

Zeyada FM, Hassan GH, Ahmed MA (2006) A generalization of a fixed point theorem due to Hitzler and Seda in dislocated quasi-metric spaces. Arab J Sci Eng Sect A 31(1):111-114

\section{Submit your manuscript to a SpringerOpen ${ }^{\circ}$ journal and benefit from:}

- Convenient online submission

- Rigorous peer review

- Immediate publication on acceptance

- Open access: articles freely available online

- High visibility within the field

- Retaining the copyright to your article

Submit your next manuscript at $>$ springeropen.com 